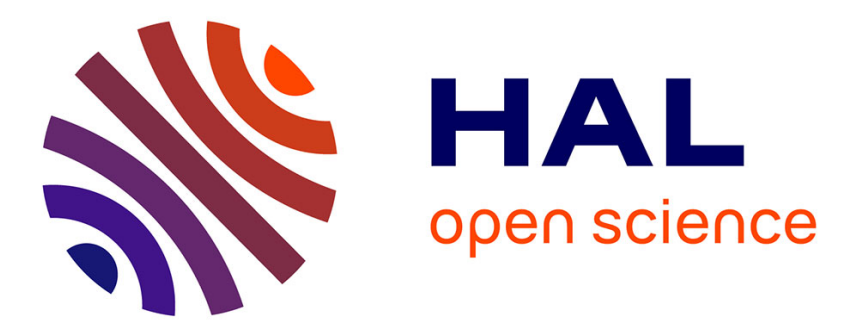

\title{
Centrifugal partition chromatography as a fractionation tool for the analysis of lignocellulosic biomass products by liquid chromatography coupled to mass spectrometry
}

Alexis Dubuis, Agnès Le Masle, Ludovic Chahen, Emilie Destandau, Nadège

Charon

\section{- To cite this version:}

Alexis Dubuis, Agnès Le Masle, Ludovic Chahen, Emilie Destandau, Nadège Charon. Centrifugal partition chromatography as a fractionation tool for the analysis of lignocellulosic biomass products by liquid chromatography coupled to mass spectrometry. Journal of Chromatography A, 2019, 1597, pp.159-166. 10.1016/j.chroma.2019.03.031 . hal-02184672

\section{HAL Id: hal-02184672 \\ https://hal-ifp.archives-ouvertes.fr/hal-02184672}

Submitted on 16 Jul 2019

HAL is a multi-disciplinary open access archive for the deposit and dissemination of scientific research documents, whether they are published or not. The documents may come from teaching and research institutions in France or abroad, or from public or private research centers.
L'archive ouverte pluridisciplinaire HAL, est destinée au dépôt et à la diffusion de documents scientifiques de niveau recherche, publiés ou non, émanant des établissements d'enseignement et de recherche français ou étrangers, des laboratoires publics ou privés. 


\title{
Centrifugal partition chromatography as a fractionation tool for the analysis of lignocellulosic biomass products by liquid chromatography coupled to mass spectrometry
}

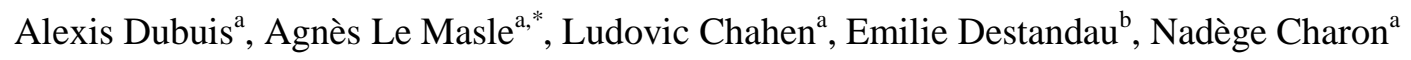 \\ ${ }^{a}$ IFP Energies nouvelles, Rond-point de l'échangeur de Solaize, BP 3, 69360 Solaize, France \\ ${ }^{b}$ Institut de Chimie Organique et Analytique, Université d'Orléans, CNRS UMR 7311, 2 Rue de Chartres, 45067 \\ Orléans, France \\ ${ }^{*}$ Corresponding author. E-mail address: agnes.le-masle@ @ifpen.fr (A. Le Masle)
}

\section{Abstract}

The conversion of lignocellulosic biomass into biofuels and bio-products leads to oxygenated matrices having a wide range of polarities and molecular weights. A complete analytical characterization of these complex mixtures is necessary to improve conversion processes. In this study, an innovative centrifugal partition chromatography (CPC) protocol was developed to fractionate aqueous biomass samples with a MTBE-water solvent system, by mixing elution, displacement and extrusion modes in the same run. This new protocol was validated on model molecules and applied to the water soluble phase of a fast pyrolysis bio-oil. It demonstrated a promising separation with a relevant selectivity on the most significant chemical families of biomass samples: carbohydrates, furans, carboxylic acids and phenols. CPC fractions of the sample were collected and analyzed comprehensively by HPLC-UV/MS (with ESI negative and positive ionization modes). This CPC x LC approach allowed more accurate attributions on the 217 peaks detected. The use of different detection modes gave a complete view of the water soluble phase of a fast pyrolysis bio-oil through 2D maps. Molecular characterization was enhanced by independent information: CPC retention time, LC retention time, UV and MS spectra. Concomitance of these different chemical information is of precious help for unambiguous identification.

Highlights:

- $\quad$ CPC chemical fractionation of the water soluble phase of a fast pyrolysis bio-oil

- Mix of elution, displacement and extrusion modes

- Prediction of the mobile phase pH for an optimized separation

- Off-line comprehensive two-dimensional liquid chromatography

- Multi-detection 'fingerprints' of the sample

Keywords: lignocellulosic biomass, fast pyrolysis bio-oil, centrifugal partition chromatography, mass spectrometry, off-line comprehensive two-dimensional liquid chromatography.

\section{Introduction}

Resources alternatives to fossil fuels will be needed in the near future to diversify energy sources and meet the rising global demand. Among renewable solutions, the conversion of lignocellulosic biomass into biofuels and bio-based chemicals is a promising way under development [1-3]. The raw material 
of biomass contains three major constituents: cellulose and hemicelluloses, respectively linear and branched polysaccharides, and the lignin made of aromatic macromolecules [4,5]. Products resulting from biochemical or thermochemical conversion processes are complex matrices with many oxygenated chemical functions like carboxylic acids, phenols, aldehydes, ketones, carbohydrates, furans or alcohols. In general, these matrices have special features: a wide range of polarities and molecular weights, acidity and heat sensitivity [6]. In this context, analytical tools have a key role to play in order to better understand the composition and reactivity of biomass products.

Gas chromatography (GC) and comprehensive two-dimensional gas chromatography (GC x GC) were extensively used to identify and quantify volatile oxygenated compounds [7,8]. Liquid chromatography (LC) and supercritical fluid chromatography (SFC) were used to characterize compounds of higher molecular weight or thermally unstable $[9,10]$. On-line comprehensive twodimensional LC (LC x LC) was applied successfully on aqueous biomass samples to enhance the peak capacity [11,12]. These complementary separation approaches and hyphenation to high resolution mass spectrometry (MS) provided a detailed molecular characterization of biomass products. However, despite the high resolution of these systems, a large part of compounds remains unknown due to co-elutions and the complexity of MS spectrum [13].

A different approach consists in fractionating the sample prior to analysis in order to raise co-elutions and improve its analysis with the existing techniques. Liquid-liquid extraction (LLE) has been widely used since it allows a simple separation by solubility differences and a full recovery of the sample with no irreversible adsorption on a solid phase. Previous studies have shown the value of a controlled LLE sample preparation to facilitate the analysis [14,15]. For instance, Kanaujia et al. demonstrated the interest of LLE to selectively classify lignocellulosic biomass degradation products into chemical families on the base of their solubility [16]. Such fractionation is of precious help to guide the identification of unknown products, or to choose between different structures for the same chemical formula.

To go further on, centrifugal partition chromatography (CPC) is a liquid-liquid separation technique based on solubility differences with a much higher efficiency than LLE. A solubility fractionation by CPC seems relevant since it provides a different selectivity than LC in reversed phase. Furthermore, CPC is compatible with heat sensitive compounds (performed at room temperature) and prevents sample lost (no irreversible adsorption on a solid phase). The CPC column is constituted by hundreds of extraction cells connected in series, filled with a stationary phase retained by the application of a centrifugal force while a mobile phase is pumped through it $[17,18]$. CPC was initially developed for the purification of natural products and then extended to other areas of application, including biorefining products analysis [19-21]. Recently, Le Masle et al. demonstrated the interest of a first dimensional CPC separation applied on a biomass sample prior to analysis by reversed phase liquid chromatography (RPLC) [22]. However, even if this approach allows to raise co-elutions, the characterization still remains difficult since the resulting 2D chromatograms shows no real chemical information or organization.

CPC can also be used to separate mixture of organic acids or phenols according to their $\mathrm{pH}$ in displacement mode, better known as 'pH zone refining'. This technique consists in controlling the $\mathrm{pH}$ of the phases by adding additives to the solvent system [23-26]. Since biomass samples contain ionizable organic acids and phenols, CPC in displacement mode may be an interesting way of fractionation. Hamzaoui et al. proposed the CPC isolation of phenolic compounds from a crude bark extract using an interesting mix of elution, displacement and extrusion modes [27]. However the $\mathrm{pH}$ zone refining uses additives like salts at relatively high concentrations, which is detrimental for MS 
analysis $[28,29]$. Moreover this mode is usually dedicated to products belonging to the same chemical class with close pKa and fractionation experiments are very time and solvent consuming [30].

In the present study, we propose to use CPC as a first dimension to fractionate any aqueous biomass sample composed of both neutral and ionizable compounds using an innovative operating combination of elution, displacement and extrusion modes. The objective is to develop a CPC protocol able to produce fractions representative of oxygenated chemical class and analyzable by RPLC/MS. The proposed method allows especially the fractionation of ionizable species without adding additives commonly used in conventional $\mathrm{pH}$ zone refining. This new CPC fractionation approach hyphenated to LC/MS was applied to the water soluble phase of a fast pyrolysis bio-oil.

\section{Experimental}

\subsection{Materials}

Methyltertiobutylether (MTBE), methanol, acetonitrile were HPLC grade purchased from VWR (Fontenay sous Bois, France). Sodium hydroxide solution ( $\mathrm{NaOH}, 0.5 \mathrm{~mol} / \mathrm{L}$ ) was obtained from Carlo-Erba reagent (Val de Reuil, France). Deionized water was produced by a Milli-Q water purifier (Millipore SAS, Molsheim, France). Formic acid, acetic acid, 5-(hydroxymethyl)furan-2-carbaldehyde (5-HMF), (2R,3S,4S,5R,6S)-2-(hydroxymethyl)-6-[2-(hydroxymethyl)phenoxy]oxane-3， 4,5-triol (salicin), (E)-3-(4-hydroxy-3-methoxy-phenyl)prop-2-enoic acid (ferulic acid), 2-methoxyphenol (guaiacol) and (2E)-3-phenylprop-2-enal (cinnamaldehyde) were purchased from Sigma-Aldrich (Saint-Quentin-Fallavier, France). 5-HMF, salicin, ferulic acid, guaiacol and cinnamaldehyde were used to prepare a model mix at $5 \mathrm{~g} / \mathrm{L}$ in methanol. This solution was then diluted to $250 \mathrm{mg} / \mathrm{L}$ in deionized water to prepare the model mix 1 and 2 (designated by MM1 and MM2). Acetic acid was added to MM2 at $3.2 \mathrm{~g} / \mathrm{L}$.

The sample used in this study was the water soluble phase of a fast pyrolysis bio-oil from softwood sawdust provided by IFP Energies nouvelles (Solaize, France). The sample was filtered prior to CPC separation using INTERCHIM PTFE syringe filters (UPTIDISC, pore size: $0.20 \mu \mathrm{m}$, diameter: $4 \mathrm{~mm}$, Montluçon, France).

\section{2. $C P C$ instrumentation}

CPC experiments were performed on a SCPC100 associated to a Spot Prep II from Armen Instrument, France (Gilson Purification, USA). The column used has an exact volume of $131 \mathrm{~mL}$ and the apparatus a total of $20 \mathrm{~mL}$ of dead volume. The system was equipped with a quaternary pump, an automatic sample injection valve with a $5 \mathrm{~mL}$ sample loop, a diode array detector (acquisition wavelength range: $200-400 \mathrm{~nm}$ ) and a fraction collector. All data were managed using the Armen Glider CPC Control Software. Experiments were conducted at room temperature $\left(20 \pm 2{ }^{\circ} \mathrm{C}\right)$.

\subsection{Preparation of solvent system}

The biphasic solvent system was prepared by mixing in a separatory funnel MTBE and water with a $50: 50$ volumetric ratio. The funnel was agitated, left 5 minutes for equilibration and the two phases were separated. The aqueous phase was selected as the mobile phase and the organic phase as the stationary phase (descending mode). The organic phase was kept for the separation (A). The $\mathrm{pH}$ of the mobile phase was adjusted by adding sodium hydroxide solution $(0.5 \mathrm{~mol} / \mathrm{L})$ to the aqueous phase under the control of a combined pH electrode (Fisher Scientific, Illkirch, France). Three bottles of mobile phase were prepared: the first without adding sodium hydroxide to the aqueous phase (B), the second at the $\mathrm{pH}$ predicted by the model (C) and the third at $\mathrm{pH} 12$ (D). 
129 CPC method conditions are described in Table 1.

130 The stationary phase ratio was measured at $77 \%$. UV signal was monitored at $254 \mathrm{~nm}$. Fractions were collected every minute during the 45 minutes of the method and numbered consequently from 1 to 45 . A rinsing method was used in ascending mode to neutralize residual hydroxide ions from the column. A pH between 7 and 8 was expected at the fraction collector by the end of the rinsing step.

\subsection{Determination of partition coefficient}

The partition coefficient $\mathrm{K}$ corresponds to the ratio of the solute concentrations $[\mathrm{S}]$ in the organic and in the aqueous phases (Equation 1):

$K=\frac{[S] \text { org }}{[S] a q}($ Equation 1$)$

$\mathrm{K}$ was measured on model molecules in the MTBE-water system. The biphasic system was prepared as described in part $2.3 .5 \mathrm{~mL}$ of each phase and approximately $100 \mathrm{mg}$ of standard were added to a 20 $\mathrm{mL}$ flask. After vigorous shaking and 5 minutes of equilibration, $1 \mathrm{~mL}$ of each phase was transferred into vials. The upper phase was evaporated under nitrogen flux and $1 \mathrm{~mL}$ of methanol was added to the vials. Upper and lower phase were analyzed by HPLC-UV (method described in part 2.7).

In this study, the constant used to define the partition equilibrium of acid on its HA form between organic and aqueous phases was defined as $\mathrm{K}_{\mathrm{D}}$ (Equation 2):

$K_{D}=\frac{[H A] \text { org }}{[H A] a q}($ Equation 2)

The $\mathrm{pH}$ of the aqueous phase was measured to determine the proportion of neutral and ionic species for $\mathrm{K}_{\mathrm{D}}$ determination.

\subsection{Off-line comprehensive two-dimensional CPC $x$ LC analysis}

A total of $45 \mathrm{CPC}$ fractions were collected. $\mathrm{pH}$ was measured from fractions 1 to 35 with a combined $\mathrm{pH}$ electrode. All fractions were then evaporated to dryness under nitrogen and dissolved into $1 \mathrm{~mL}$ of methanol (to the same dilution factor for elution and extrusion). Fractions 6 and 7 were filtered using INTERCHIM PTFE syringe filters because of the presence of some insoluble particles (UPTIDISC, pore size: $0.20 \mu \mathrm{m}$, diameter: $4 \mathrm{~mm}$, Montluçon, France). The sample was prepared in the same conditions as CPC fractions for 1D LC chromatograms.

\subsection{High performance liquid chromatography coupled to mass spectrometry}

HPLC analyses were performed on a Shimadzu LC 20AD (Kyoto, Japan) composed of a binary pump, a SIL 20AC autosampler $\left(10^{\circ} \mathrm{C}\right)$, a CTO $20 \mathrm{AC}$ column oven $\left(30^{\circ} \mathrm{C}\right)$, a SPD M20A UV detector and a CBM 20A system controller. The injection volume was $5 \mu \mathrm{L}$. Separation was achieved on a Kinetex C18 column ( $150 \times 3 \mathrm{~mm}, 2.6 \mu \mathrm{m}$, Phenomenex, Le Pecq, France) at $30^{\circ} \mathrm{C}$. Mobile phases used were water (A1) and acetonitrile (B1) both acidified with $0.025 \%$ (v:v) of formic acid. The separation was performed at $0.6 \mathrm{~mL} / \mathrm{min}$ with the following elution gradient: $0-6 \mathrm{~min}(100 \% \mathrm{~A} 1), 6-26 \mathrm{~min}$ (from $0 \% \mathrm{~B} 1$ to $40 \% \mathrm{~B} 1$, normalized elution gradient slope of $2.2 \%), 26-26.1 \mathrm{~min}(100 \% \mathrm{~A} 1)$ and $26.1-$ $32 \mathrm{~min}(100 \% \mathrm{~A} 1)$. The dwell time was measured at $1.1 \mathrm{~min}$. UV signal was measured on the wavelength range $200-400 \mathrm{~nm}$ (Deuterium lamp, cell temperature of $30{ }^{\circ} \mathrm{C}$, sampling frequency of $12.5 \mathrm{~Hz}$ and time constant of $0.080 \mathrm{~s}$ ). When only a UV detection signal was desired, fractions 1 to 35 
can be directly injected by HPLC without evaporation step. MS detection was performed on a Shimadzu 2020 simple quadrupole. The flow rate was reduced to $150 \mu \mathrm{L} / \mathrm{min}$ for MS detection using a post UV T-split. An Electrospray Ionization (ESI) source was used in negative and positive modes with an interface voltage of $-4.5 \mathrm{kV}$ and $+4.5 \mathrm{kV}$ respectively. Drying and nebulizing gas flow rates were respectively $15 \mathrm{~L} / \mathrm{min}$ and $1.5 \mathrm{~L} / \mathrm{min}$. Temperatures were set to $200{ }^{\circ} \mathrm{C}$ for the heat block and 250 ${ }^{\circ} \mathrm{C}$ for the desolvation line. The scan range was set to $100-800 \mathrm{~m} / \mathrm{z}$. Reproducibility of retention times and MS sensitivity were checked with standards injected regularly during sequences (Supplementary material). Data acquisition and treatment were managed with Shimadzu software Labsolution.

\section{Results and discussion}

\subsection{LC/MS analysis of the water soluble phase of a fast pyrolysis bio-oil}

The aqueous biomass sample was analyzed by HPLC-UV/MS using generic conditions to have a first description of its complexity. Chromatograms obtained with UV and MS detection are presented in Figure 1.

The proposed reversed phase separation coupled to mass spectrometry offers a first molecular description of the sample. This direct analysis allows the detection of approximately 70 peaks with UV detection, 55 with ESI positive and 60 with ESI negative. All modes included, 139 peaks were counted manually in Figure 1, considering peaks with a signal-to-noise ratio greater than 3 (table in Supplementary material). UV and MS detections provide complementary profiles of the sample, with maxima positioned at different times of the chromatogram. However, the chromatograms present many co-elutions and are therefore difficult to process. A first dimensional separation is required to increase the peak capacity and simplify chromatograms.

\subsection{CPC separation of the aqueous biomass sample}

\subsubsection{Selection of the solvent system}

The biphasic system is essential in CPC because it represents the mobile and the stationary phases. In this study, the objective is to fractionate the entire sample rather than extracting a single compound, using elution and displacement modes. To do so, the biphasic system MTBE-water appears to be well adapted for many reasons. Firstly, this system is very stable and commonly used for both classical elution and $\mathrm{pH}$ zone refining applications [23,27]. Moreover, MTBE is able to extract a large number of organic species present in biomass products, including acids and phenolic compounds [31]. The organic phase is preferentially used as a stationary phase to retain ionizable chemical compounds. Additionally, the mobile phase has to be aqueous to modify the $\mathrm{pH}$ during the separation and ionize compounds retained at the head of the CPC column. Thus, compounds will be eluted with the aqueous mobile phase under their ionic form, depending on their pKa. This descending mode configuration is better for the system stability according to Ito [17] and is more suitable for coupling with RPLC (production of directly analyzable aqueous fractions).

\subsubsection{Fractionation strategy}

In a classical CPC experiment with elution and extrusion modes, the separation relies only on the partition coefficient $(\mathrm{K})$ of solutes in the biphasic system. In accordance with CPC usual recommendations, $\mathrm{K}$ should be within the range $0.5-3$ in the elution zone to maximize peaks resolution and reduce peaks broadening [17]. The partition coefficient of a solute can be used to calculate the retention time, knowing CPC operating parameters (column volume, stationary phase retention ratio, flow rate) [18]. In this way, common chemical families present in biomass samples can 
be positioned on a CPC chromatogram. Thus, in classical CPC elution/extrusion modes in descending mode, carbohydrates and their derivatives are eluted in the first fractions, close to the dead volume because of their high affinity to water. Then, furans and other small polar compounds are eluted $(0.5<$ $\mathrm{K}<1.5$ ). For these chemical families, the elution mode allows an excellent fractionation. However, the less polar compounds like carboxylic acids, phenols or aldehydes or hybrids compounds are eluted, with more dispersed peaks in the rest of the elution $(1.5<\mathrm{K}<3)$, and mainly in the extrusion fractions $(\mathrm{K}>3)$ without clear chemical fractionation. The proposed strategy in Figure 2 consists in adding a displacement mode to force ionizable species like carboxylic acids and phenols to elute, in a limited zone of elution, after the furans and before the extrusion mode.

The order of separation of the displaced compounds should mainly follow their $\mathrm{pKa}$. It is important to notice that, for the rest of the publication, 'hybrid' species like phenolic carboxylic acids are considered as carboxylic acids since their lower pKa corresponds to the carboxylic acid function.

In this strategy, the selectivity between carboxylic acids and phenols has to be optimized and the key factor is the $\mathrm{pH}$ of the mobile phase at the intermediate step. This $\mathrm{pH}$ needs to be high enough to neutralize the total sample acidity and consequently, to allow the complete elution of carboxylic acids. However, if the chosen $\mathrm{pH}$ is too high, phenol will be ionized also and may co-elute with carboxylic acids, losing the interest of an intermediate step. Finally, the intermediate $\mathrm{pH}$ depends of the total acidity of the sample and has to be calculate prior to the CPC protocol.

\subsubsection{Modelling approach}

In this section are presented the main hypothesis considered to predict the elution of ionizable species during the displacement mode. The resulting model allows to estimate the sodium hydroxide quantity necessary to elute all acidic compounds of the sample within a limited time of elution. The corresponding operating parameter is the intermediate $\mathrm{pH}$ of the mobile phase, manually adjusted using a combined $\mathrm{pH}$ electrode. An exhaustive study of the phenomena at the liquid-liquid interface in pH zone refining was proposed by Kotland et al. [24]. Herein, the model developed does not take into account all hydrodynamic aspects involved. For the calculations, the CPC column was assimilated to a succession of LLE cells connected in series, considering a limited number of 100 theoretical plates, enough to have an accurate prediction of species behavior using the displacement mode. Each cell can be represented by the situation described in Figure 3.

In Figure 3, all ionizable species $(3<\mathrm{pKa}<11)$ are symbolized by $\mathrm{HA}$ for the acidic form and $\mathrm{A}^{-}$for the conjugate base. The $\mathrm{A}^{-}$form is considered as totally insoluble in the organic phase. Regarding the pKa of carboxylic acids $(3<\mathrm{pKa}<4)$ and considering that the $\mathrm{pH}$ intermediate would be certainly superior to 7 , the reaction in the aqueous phase between the hydroxide ion $\mathrm{HO}^{-}$and $\mathrm{HA}^{\mathrm{A}}$ is supposed total, with a constant $\mathrm{K}_{\mathrm{aq}}$ (Equation 3):

$K_{a q}=\frac{[A-] a q}{[H A] a q *[H O-] a q}($ Equation 3)

The equilibrium defined by the $\mathrm{K}_{\mathrm{D}}$ constant is displaced by the consumption of $\mathrm{HA}_{\mathrm{aq}}$ which is then transferred from cell to cell in its $\mathrm{A}^{-}{ }_{\mathrm{aq}}$ form. Before starting this transfer of HA from the organic to the aqueous phase, the complete acidity of the sample introduced in the first cell needs to be neutralized. Therefore, a titration of the total amount of acid is necessary. However, small organic acids (formic acid, acetic acid) which are common degradation products in biomass samples do not require neutralization since they are no longer present in the CPC column during the intermediate step $(\mathrm{K}<$ 1.5). Consequently, these acids should be quantified independently and subtracted from the total acidity. The acid concentration obtained $[\mathrm{HA}]_{\mathrm{aq}}$ is then implemented in the model. 
To calculate the quantity of solute transferred, others CPC parameters are needed in the model: the stationary phase retention ratio, the column volume, the injection volume, and the flow rate. The $\mathrm{K}_{\mathrm{D}}$ was determined for the biphasic system MTBE-water using the LC-UV method described in section 2.7 within the range $0.6-940$ (respectively for the pyruvic and the ferulic acids, commonly present in aqueous biomass samples). By fixing all others parameters, the two limits $K_{D}=0.5$ and $K_{D}=2000$ were evaluated in the model, and the $K_{D}$ demonstrated a limited influence on the peak width. The $K_{D}$ value of the ferulic acid was considered by default for the model. In practice, the model uses a spreadsheet and the results are displayed with graphical representation of concentration profiles of each specie as a function of the time, at different theoretical plates (corresponding to different positions in the column). The elution of acidic compounds can be monitored with species A- at the final theoretical plate. The $\mathrm{pH}$ of the mobile phase can be finely adjusted by the user to control the width of the peak. In this study, a peak width of 10 minutes was fixed as the optimal response for the elution of acids, corresponding to the duration of the intermediate step.

\subsection{Validation of the fractionation protocol}

\subsubsection{Model molecules}

The feasibility of the CPC protocol was validated on model molecules representative of the targeted chemical families. In biomass samples, there are numerous carboxylic acids species and they can be present in various concentrations. The acetic acid is one of the main degradation product of lignocellulosic biomass and its concentration is often largely superior to other carboxylic acids. In the water soluble phase of a pyrolysis bio-oil, the acetic acid was quantified at $3.4 \mathrm{~g} / \mathrm{L}$ using a LC-UV reference method at $210 \mathrm{~nm}$ [32]. Acid acetic should be eluted before the displacement zone because of its high hydrophilicity $(\mathrm{K}<1)$ and therefore should not consume hydroxides ions of the mobile phase. However, it was important to measure the influence of high concentration of acetic acid on the separation of other carboxylic acids and phenols. Two mixes containing both 5 model molecules were prepared: one without acetic acid (MM1) and one with $3.2 \mathrm{~g} / \mathrm{L}$ of acetic acid (MM2). A pH of 9.8 was predicted for the mobile phase to elute ferulic acid of MM1 and MM2 in 10 minutes. CPC chromatograms obtained for MM1 and MM2 are presented in Figure 4.

Salicin (1), hydroxymethylfurfural (2), guaiacol (4) and cinnamaldehyde (5) are eluted at the same position of chromatograms in Figure 4. Salicin is a glucoside (carbohydrate derivative) with a high solubility in water, this explains its elution within the dead volume $(6-7 \mathrm{~min})$. Hydroxymethylfurfural retention time $(9 \mathrm{~min})$ is consistent with its partition coefficient $(\mathrm{K}=0.4)$. The guaiacol is displaced at 30 minutes in the phenol zone of both chromatograms. The signal observed between 35 and 37 minutes is a disturbance due to the elution of the stationary phase during extrusion. The cinnamaldehyde is eluted at 40 minutes in the extrusion in accordance with its physicochemical properties $(\mathrm{K}>3, \mathrm{pKa}>14)$. Still, differences can be noticed between the two model mix chromatograms. The elution of acetic acid in MM2 results as anticipated in a $\mathrm{pH}$ drop of the aqueous mobile phase between 9 and 16 minutes. Acetic acid and hydroxymethylfurfural are co-eluted on the $210 \mathrm{~nm}$ CPC chromatograms provided in supplementary material. The peak of ferulic acid in MM2 is delayed and broadened compared to MM1. Thus, displacement of the ferulic acid is less efficient for MM2 because hydroxides of the mobile phase are consumed by the remaining acetic acid in the column. The peak width of 10 minutes predicted was correct for the ferulic acid, but the risk is to elute a part of acids on phenols elution window. For this reason, the $\mathrm{pH}$ of the mobile phase predicted by the model should be increased of $0.1-0.2$ point, to reduce acids peak width and prevent additional acidity coming from unknown acids of the sample in the elution zone. Acetic acid has also a positive effect on the separation by retaining more efficiently acids in CPC column head. Thereby, it avoids the elution of a small proportion of acids within the dead volume, as for MM1 in Figure 4 (see the baseline signal 
between peaks 1 and 2). The high acid content in biomass samples acts like an acid buffer for CPC first cells, leading to the situation observed for MM2. These CPC tests realized on model molecules confirmed the good chemical selectivity of the protocol.

\subsubsection{Water soluble phase of a fast pyrolysis bio-oil}

The first dimensional CPC separation was then applied on the water soluble phase of a fast pyrolysis bio-oil. First, the complex sample was fractionated by CPC using the classical elution - extrusion mode without $\mathrm{pH}$ modification of the mobile phase (chromatogram available in supplementary material) to evaluate the separation of the different solute families only based on their partition coefficient K. A promising separation was obtained by elution for the first part of the chromatogram (0 - $15 \mathrm{~min}$ ) and a band was recovered in the extrusion. In these highly retained fractions some molecules carry ionizable functions (carboxylic acids, phenols), justifying the use of the displacement mode to elute them in the empty part of the chromatogram $(15-35 \mathrm{~min})$. Thus, the acidity of the sample was estimate at $4.4 \mathrm{mmol} / \mathrm{L}$. Using the predictive model, the $\mathrm{pH}$ of the mobile phase should be 10.4 to elute acids during the intermediate step. According to the model, a pH of 10 is not enough to elute acids and a $\mathrm{pH}$ of 11 reduces the width of the acid representative peak to 3 minutes, which may lead to co-elutions. CPC fractionations at $\mathrm{pH} 10,10.5$ and 11 for the intermediate step were investigated to assess the quality of these predictions. An additional experiment was done without intermediate step (equivalent to a direct step at $\mathrm{pH}$ 12). The aim was to find the practical best intermediate $\mathrm{pH}$ for this sample. Corresponding CPC chromatograms are given in Figure 5.

CPC chromatograms are well overlaid in the elution zone $(0-17 \mathrm{~min})$, showing the repeatability of the elution fractionation. $\mathrm{pH}$ measurements in the fractions confirmed the presence of acids eluted by their partition coefficients. Two drops in $\mathrm{pH}$ are observed: the first may be the formic acid $(5-7 \mathrm{~min}$, not retained with MTBE-water solvent system) and the second is the acetic acid ( $9-17 \mathrm{~min}$, as for the model mix experiments). In the displacement zone $(17-27 \mathrm{~min})$, chromatograms are very different depending on the intermediate $\mathrm{pH}$ used. This demonstrates the interest of $\mathrm{pH}$ modifications and the key role of the intermediate step. Best separations are obtained with the intermediate $\mathrm{pH}$ of 10.5 and 11 as a band appears between 17 and 27 minutes. The tests realized at $\mathrm{pH} 10$ and without intermediate step (directly $\mathrm{pH} 12$ ) confirm the existence of an optimal $\mathrm{pH}$. In fact, with an intermediate $\mathrm{pH}$ of 10 , as predicted by the model, acidic compounds are not eluted in the band $(17-27 \mathrm{~min})$ but the peak at 28 minutes is more important than those at $\mathrm{pH} 10.5$ and 11. The reason is an insufficient amount of hydroxide ions supplied by the mobile phase to neutralize the acidity of the sample in the first cells of the CPC, which delays the displacement of all ionizable compounds to the $\mathrm{pH} 12$ step. Though, the opposite effect is observed with a direct step at $\mathrm{pH} 12$ (experiment without intermediate step), as the peak at 27 minutes is much higher, because it contains the majority of ionizable products. The experiment without intermediate step shows the presence of a peak at 20 minutes eluted in the displacement zone with its partition coefficient (peak more dispersed). In the phenols zone, a peak constituted of a less ionizable chemical family is present in all chromatograms after the main peak (between 29 and 32 minutes). No UV peak is detected in the extrusion, so $\mathrm{pH} 12$ was enough to elute the majority of ionizable products retained in the stationary phase. The CPC fractionation was applied successfully on the water soluble phase of a pyrolysis bio-oil. The intermediate $\mathrm{pH}$ of 10.5 provides the best separation considering the band spreading of the intermediate step, in accordance with model prediction. Therefore, $\mathrm{pH} 10.5$ fractions were selected to perform the second dimensional separation by RPLC-UV/MS.

\subsection{Off-line comprehensive two-dimensional CPC x LC analysis using UV and MS detections}


To go further in the molecular characterization of the sample, a second LC separation was performed comprehensively on CPC fractions. This two-dimensional approach benefits from the chemical organization of CPC and the high resolving power of HPLC, which has already demonstrated a promising separation on the initial sample (Figure 1). The limited use of additive with the displacement mode allows coupling with mass spectrometry. The objective of this discussion is to show the interest of using the CPC protocol as a first dimension in an off-line coupling with HPLCUV/MS, taking advantage of the complementarity of the separation modes and three different detections: UV, MS (ESI negative, ESI positive). An evaporation step was performed on CPC fractions to improve MS detected signals. The 2D contour plots obtained are presented in Figure 6.

2D maps were represented using a home-made software also used to detect all local maxima with a minimum intensity equal to 3 times the signal to noise ratio. A total of 217 peaks are reported combining the three detection modes after manual checking to remove integrations due to the LC elution gradient (vertical lines in Figure 6). In comparison with the 139 peaks detected by 1D LC, this high number of detected peaks demonstrates the need for a first dimensional CPC separation and its complementarity with HPLC. Early CPC fractions (5 - $7 \mathrm{~min}$ ) have a band in the UV map which remains difficult to process like in the 1D LC chromatogram. However, the elution of this highly water-soluble fraction (certainly mainly carbohydrates derivatives) greatly improved the rest of the map. In this way, many co-elutions were raised with the CPC separation, especially in the displacement zone. Some peaks were detected by MS in the extrusion, which could not be anticipated from the CPC-UV chromatogram (Figure 5). The absence of UV response in the range $200-400 \mathrm{~nm}$ reduces possible propositions for the chemical family of these unknown products (terpenoids, fatty acids, sterols, cutins...). 2D maps simplify the comparison of the three detections used, providing strong arguments for identification. On the 217 molecules, 112 were detected by UV, 99 by ESI negative and 70 by ESI positive (table of comparison provided in the Supplementary material). The multi-detection proposed provide additional arguments for qualitative analysis. This chemical information along with the CPC retention time are helpful for structural elucidation. An identification was proposed when molecules were detected with at least two modes and after a comparison with UV and MS standards spectrum (Table 2).

Molecules identified are mainly phenolic compounds from the displacement zone. The physicochemical properties of these products were compared to their CPC retention times to assess the chemical selectivity of the CPC fractionation. 5-HMF is logically eluted in the elution zone (11 min) in accordance with its negative $\log \mathrm{P}(-0.1)$. Phenolic acids with a pKa of approximately 4 are eluted in fractions 21 and 22. In these first fractions, the separation is clearly driven by the acid function (pKa). From fractions 23 to 26, products are also mainly eluted in the ascending order of their pKa. However, hydrophobicity plays a major role on the separation of phenols which are not carrying an acid function. For instance, syringaldehyde $(\mathrm{pKa}=7.2)$ is eluted in fraction 22 probably because of its low hydrophobicity $(\log \mathrm{P}=1.1$ ). Peaks more dispersed on the CPC axis of the $2 \mathrm{D}$ map can be assigned to compounds eluted by their partition coefficient rather than displacement. 3,4-dihydroxybenzaldehyde ( $\log \mathrm{P}=1.1$ ) is the best example as it is eluted from fractions 16 to 24 on the UV map of Figure 6 (also visible on the CPC chromatogram of Figure 5). The elution order of phenols combined the properties $\mathrm{pKa}-\log \mathrm{P}$ and is therefore more difficult to understand than acids. These few products confirm the interest of the CPC protocol to enhance identification of a complex sample. The 2D approach combined with different detection modes is a powerful tool, providing complementary fingerprints of samples.

\section{Conclusion}


A CPC protocol was developed to fractionate an aqueous biomass sample by chemical family with a MTBE-water solvent system, by mixing elution, displacement and extrusion modes. A modeling approach was used to determine the optimal $\mathrm{pH}$ for the aqueous mobile phase in the displacement zone. Protocol and model were validated on model molecules and applied to the water soluble phase of a fast pyrolysis bio-oil. The first dimensional CPC demonstrated a promising separation with a relevant selectivity on the most significant chemicals families of biomass samples: carbohydrates, furans, carboxylic acids and phenols. CPC fractions of the complex sample were collected and analyzed comprehensively by LC-UV/MS (ESI negative and positive). This two-dimensional approach allowed the detection of 217 peaks with more accurate attributions. The use of different detection modes gives a complete view of the sample through 2D contour plots. Molecular characterization is enhanced by CPC retention time, LC retention time, UV and MS spectra. 2D maps representations by chemical classes can also be used as fingerprints to compare samples coming from various feedstocks or processes.

\section{Acknowledgments}

The authors thank Pascal Duchene and Damien Leinekugel Le Cocq from IFP Energies nouvelles for their help with 2D data management and modelling calculations.

\section{References}

[1] A.K. Chandel, V.K. Garlapati, A.K. Singh, F.A.F. Antunes, S.S. da Silva, The path forward for lignocellulose biorefineries: Bottlenecks, solutions, and perspective on commercialization, Bioresour. Technol. 264 (2018) 370-381. https://doi.org/10.1016/j.biortech.2018.06.004.

[2] P.R. Seidl, A.K. Goulart, Pretreatment processes for lignocellulosic biomass conversion to biofuels and bioproducts, Current Opinion in Green and Sustainable Chemistry 2 (2016) 48-53. https://doi.org/10.1016/j.cogsc.2016.09.003.

[3] P. Gallezot, Conversion of biomass to selected chemical products, Chem. Soc. Rev. 41 (4) (2012) 1538-1558. https://doi.org/10.1039/c1cs15147a.

[4] A. Brandt, J. Gräsvik, J.P. Hallett, T. Welton, Deconstruction of lignocellulosic biomass with ionic liquids, Green Chem. 15 (3) (2013) 550. https://doi.org/10.1039/c2gc36364j.

[5] P. McKendry, Energy production from biomass (part 1): overview of biomass, Bioresour. Technol. 83 (2002) 37-46.

[6] M. Staš, D. Kubička, J. Chudoba, M. Pospíšil, Overview of Analytical Methods Used for Chemical Characterization of Pyrolysis Bio-oil, Energy Fuels 28 (1) (2013) 385-402. https://doi.org/10.1021/ef402047y.

[7] P.K. Kanaujia, Y.K. Sharma, M.O. Garg, D. Tripathi, R. Singh, Review of analytical strategies in the production and upgrading of bio-oils derived from lignocellulosic biomass, Journal of Analytical and Applied Pyrolysis 105 (2014) 55-74. https://doi.org/10.1016/j.jaap.2013.10.004.

[8] B. Du, L.N. Sharma, C. Becker, S.-F. Chen, R.A. Mowery, G.P. van Walsum, C.K. Chambliss, Effect of varying feedstock-pretreatment chemistry combinations on the formation and accumulation of potentially inhibitory degradation products in biomass hydrolysates, Biotechnol. Bioeng. 107 (3) (2010) 430-440. https://doi.org/10.1002/bit.22829.

[9] J. Crepier, A. Le Masle, N. Charon, F. Albrieux, S. Heinisch, Development of a supercritical fluid chromatography method with ultraviolet and mass spectrometry detection for the characterization of biomass fast pyrolysis bio oils, J. Chromatogr. A 1510 (2017) 73-81. https://doi.org/10.1016/j.chroma.2017.06.003.

[10] T.M. Jarrell, C.L. Marcum, H. Sheng, B.C. Owen, C.J. O'Lenick, H. Maraun, J.J. Bozell, H.I. Kenttämaa, Characterization of organosolv switchgrass lignin by using high performance liquid 
chromatography/high resolution tandem mass spectrometry using hydroxide-doped negative-ion

mode electrospray ionization, Green Chem 16 (5) (2014) 2713-2727. https://doi.org/10.1039/C3GC42355G.

[11] A. Le Masle, D. Angot, C. Gouin, A. D'Attoma, J. Ponthus, A. Quignard, S. Heinisch, Development of on-line comprehensive two-dimensional liquid chromatography method for the separation of biomass compounds, J. Chromatogr. A 1340 (2014) 90-98. https://doi.org/10.1016/j.chroma.2014.03.020.

[12] D. Tomasini, F. Cacciola, F. Rigano, D. Sciarrone, P. Donato, M. Beccaria, E.B. Caramão, P. Dugo, L. Mondello, Complementary analytical liquid chromatography methods for the characterization of aqueous phase from pyrolysis of lignocellulosic biomasses, Anal. Chem. 86 (22) (2014) 11255-11262. https://doi.org/10.1021/ac5038957.

[13] M. Staš, J. Chudoba, D. Kubička, J. Blažek, M. Pospíšil, Petroleomic Characterization of Pyrolysis Bio-oils: A Review, Energy Fuels 31 (10) (2017) 10283-10299. https://doi.org/10.1021/acs.energyfuels.7b00826.

[14] Y. Wei, H. Lei, L. Wang, L. Zhu, X. Zhang, Y. Liu, S. Chen, B. Ahring, Liquid-Liquid Extraction of Biomass Pyrolysis Bio-oil, Energy Fuels 28 (2) (2014) 1207-1212. https://doi.org/10.1021/ef402490s.

[15] S. Ren, X.P. Ye, A.P. Borole, Separation of chemical groups from bio-oil water-extract via sequential organic solvent extraction, Journal of Analytical and Applied Pyrolysis 123 (2017) 30-39. https://doi.org/10.1016/j.jaap.2017.01.004.

[16] P.K. Kanaujia, D.V. Naik, D. Tripathi, R. Singh, M.K. Poddar, L.S.K. Konathala, Y.K. Sharma, Pyrolysis of Jatropha Curcas seed cake followed by optimization of liquid $\square$ liquid extraction procedure for the obtained bio-oil, Journal of Analytical and Applied Pyrolysis 118 (2016) 202224. https://doi.org/10.1016/j.jaap.2016.02.005.

[17] Y. Ito, Golden rules and pitfalls in selecting optimum conditions for high-speed counter-current chromatography, Journal of Chromatography A 1065 (2) (2005) 145-168. https://doi.org/10.1016/j.chroma.2004.12.044.

[18] A. Berthod, K. Faure, Separations with a Liquid Stationary Phase: Countercurrent Chromatography or Centrifugal Partition Chromatography, Analytical Separation Science (2015) 1177-1206. https://doi.org/10.1002/9783527678129.assep046.

[19] D.P. Ward, M. Cárdenas-Fernández, P. Hewitson, S. Ignatova, G.J. Lye, Centrifugal partition chromatography in a biorefinery context: Separation of monosaccharides from hydrolysed sugar beet pulp, J. Chromatogr. A 1411 (2015) 84-91. https://doi.org/10.1016/j.chroma.2015.08.006.

[20] M. Bojczuk, D. Żyżelewicz, P. Hodurek, Centrifugal partition chromatography - A review of recent applications and some classic references, J. Sep. Sci. 40 (7) (2017) 1597-1609. https://doi.org/10.1002/jssc.201601221.

[21] C.-S. Lau, K.A. Bunnell, E.C. Clausen, G.J. Thoma, J.O. Lay, J. Gidden, D.J. Carrier, Separation and purification of xylose oligomers using centrifugal partition chromatography, J. Ind. Microbiol. Biotechnol. 38 (2) (2011) 363-370. https://doi.org/10.1007/s10295-010-0799-1.

[22] A. Le Masle, S. Santin, L. Marlot, L. Chahen, N. Charon, Centrifugal partition chromatography a first dimension for biomass fast pyrolysis oil analysis, Analytica Chimica Acta (2018). https://doi.org/10.1016/j.aca.2018.04.040.

[23] Y. Ito, pH-zone-refining counter-current chromatography: origin, mechanism, procedure and $\begin{array}{lllllll}\text { applications, } & \text { J. } & \text { Chromatogr. } & \text { A } & 1271 & \text { (1) } & \text { (2013) }\end{array}$ https://doi.org/10.1016/j.chroma.2012.11.024.

[24] A. Kotland, S. Chollet, J.-M. Autret, C. Diard, L. Marchal, J.-H. Renault, Modeling pH-zone refining countercurrent chromatography: a dynamic approach, J. Chromatogr. A 1391 (2015) 8087. https://doi.org/10.1016/j.chroma.2015.03.005. 
[25] A. Kotland, S. Chollet, C. Diard, J.-M. Autret, J. Meucci, J.-H. Renault, L. Marchal, Industrial case study on alkaloids purification by $\mathrm{pH}$-zone refining centrifugal partition chromatography, J. Chromatogr. A 1474 (2016) 59-70. https://doi.org/10.1016/j.chroma.2016.10.039.

[26] M. Hamzaoui, J. Hubert, R. Reynaud, L. Marchal, A. Foucault, J.-H. Renault, Strong ion exchange in centrifugal partition extraction (SIX-CPE): effect of partition cell design and dimensions on purification process efficiency, J. Chromatogr. A 1247 (2012) 18-25. https://doi.org/10.1016/j.chroma.2012.05.046.

[27] M. Hamzaoui, J.-H. Renault, R. Reynaud, J. Hubert, Centrifugal partition extraction in the pHzone-refining displacement mode: an efficient strategy for the screening and isolation of biologically active phenolic compounds, J. Chromatogr. B Analyt. Technol. Biomed. Life Sci. 937 (2013) 7-12. https://doi.org/10.1016/j.jchromb.2013.07.024.

[28] M.C. García, The effect of the mobile phase additives on sensitivity in the analysis of peptides and proteins by high-performance liquid chromatography-electrospray mass spectrometry, J. Chromatogr. B Analyt. Technol. Biomed. Life Sci. 825 (2) (2005) 111-123. https://doi.org/10.1016/j.jchromb.2005.03.041.

[29] N.B. Cech, C.G. Enke, Practical implications of some recent studies in electrospray ionization fundamentals, Mass Spectrom. Rev. 20 (6) (2001) 362-387. https://doi.org/10.1002/mas.10008.

[30] G. Dong, J. Xu, Y. Gu, Y. Wei, A general separation method of phenolic acids using pH-zonerefining counter-current chromatography and its application to oat bran, J. Chromatogr. B Analyt. Technol. Biomed. Life Sci. 992 (2015) 36-42. https://doi.org/10.1016/j.jchromb.2015.04.024.

[31] S.-F. Chen, R.A. Mowery, V.A. Castleberry, G.P. van Walsum, C.K. Chambliss, Highperformance liquid chromatography method for simultaneous determination of aliphatic acid, aromatic acid and neutral degradation products in biomass pretreatment hydrolysates, J. Chromatogr. A 1104 (1-2) (2006) 54-61. https://doi.org/10.1016/j.chroma.2005.11.136.

[32] A. Sluiter, B. Hames, R. Ruiz, C. Scarlata, J. Sluiter, D. Templeton, and D. Crocker, Determination of Structural Carbohydrates and Lignin in Biomass: Laboratory Analytical Procedure (LAP): NREL/TP-510-42618, National Renewable Energy Laboratory (2008).

\section{Figure captions}

Figure 1: HPLC-UV/MS chromatograms of the water soluble phase of a pyrolysis bio-oil $(254 \mathrm{~nm}$, base peak chromatograms).

Figure 2: CPC fractionation strategy using a mix of elution, displacement and extrusion modes.

Figure 3: Simplified representation of liquid-liquid equilibria within each CPC cell.

Figure 4: CPC-UV chromatograms of the model mix MM1 without acetic acid and MM2 with acetic acid (254nm). 1. Salicin, 2. Hydroxymethylfurfural, 3. Ferulic acid, 4. Guaiacol, 5. Cinnamaldehyde.

Figure 5: CPC-UV chromatograms of the water soluble phase of a pyrolysis bio-oil $(254 \mathrm{~nm})$ performed at $\mathrm{pH} 10,10.5$ and 11 for the intermediate step. An experiment was performed without intermediate step. $\mathrm{pH}$ measurement in fractions are represented with dotted lines.

Figure 6: CPC $x$ LC contour plots of the water soluble phase of a pyrolysis bio-oil obtained with UV $(254 \mathrm{~nm})$ and MS detections (ESI negative, ESI positive). MS 2D maps were obtained from the base peak chromatograms.

Table 1: CPC conditions used in descending mode.

Table 2: Products identified in the water soluble phase of a pyrolysis bio-oil using UV and MS detections. pKa and $\log \mathrm{P}$ were obtained from https://chemicalize.com/\#/calculation. 